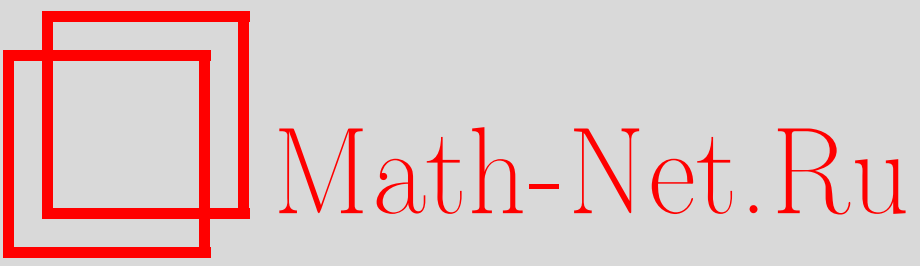

А. В. Богданов, А. С. Геворкян, А. И. Денисенко, Аналитическая аппроксимация $S$-матрицы трехчастичной коллинеарной реакции перестройки в квазиклассическом приближении, ТМФ, 1996, том 107, номер 2, 238-250

DOI: https://doi.org/10.4213/tmf1152

Использование Общероссийского математического портала Math-Net.Ru подразумевает, что вы прочитали и согласны с пользовательским соглашением

http: //www.mathnet.ru/rus/agreement

Параметры загрузки:

IP : 3.95 .254 .165

26 апреля 2023 г., $17: 51: 16$ 
ТЕОРЕТИЧЕСКАЯ

И МАТЕМАТИЧЕСКАЯ

ФИЗИКА

Том 107, № 2

май, 1996

А.В. Богданов, А.С. Геворкян, А.И. Денисенко, Г. В. Дубровский

\section{АНАЛИТИЧЕСКАЯ АППРОКСИМАЦИЯ $S$-МАТРИЦЫ ТРЕХЧАСТИЧНОЙ КОЛЛИНЕАРНОЙ РЕАКЦИИ ПЕРЕСТРОЙКИ В КВАЗИКЛАССИЧЕСКОМ ПРИБЛИЖЕНИИ}

\footnotetext{
Коллинеарная задача о трехчастичной реакции перестройки в рамках квазиклассического метода параболического уравнения Фока-Леонтовича сводится к квантовой задаче о динамике одномерного гармонического осциллятора с переменной частотой в поле внешней силы. Это дает возможность выразить матричные элементы $S$-матрицы, отвечающие каналу перестройки, через известные аналитические функции осцилляторной модели, использующие параметры потенциальной поверхности линейной конфигурации трех тел.
}

\section{1. ВВЕДЕНИЕ}

Коллинеарная модель перестройки, как наиболее простое приближение реактивного рассеяния, в течение многих лет интенсивно используется как для изучения принципиальных вопросов теории, так и для непосредственных расчетов констант скоростей химических реакций при умеренных и низких энергиях [1]. Важные результаты для этой модели в квазиклассическом приближении были получены путем траекторных расчетов с учетом комплексных путей, резонансов в рамках классической $S$-матрицы [2-4]. Следует отметить, что расчеты такого рода требуют значительных вычислительных усилий при необходимости рассмотрения широкого круга систем (как, например, в физической кинетике), варьировании потенциальных поверхностей, попытках распространения метода на задачи с большей размерностью (трехмерные, с участием большего числа тел, для химических реакций на поверхности, в конденсированной фазе и т.д.). Сказанное определяет интерес к развитию аналитических методов решения этой простейшей многомерной задачи, в рамках которых часто удается найти эффективные аналитические аппроксимации, удобные для приложений, наметить пути дальнейших обобшений. Так, например, для оценки эффективности туннельных химических реакций использовалась простая модель одномерного потенциального барьера [2-4], которая является грубым приближением к трехчастичной динамике, не учитываюшим многие физические эффекты (кривизну пути, вклады примыкающих траекторий, надбарьерные переходы). Более строгие результаты, касаюшиеся квазиклассического представления вероятности реактивного перехода в виде интеграла по пути реакции в рамках метода параболического уравнения Фока-Леонтовича, были получены в работах [5-7]. Найденное в ра- 
ботах [5-7] одномерное представление вероятности перехода может быть использовано для аналитических аппроксимаций [8], однако оно является еше довольно сложным.

В настоящей работе в рамках метода параболического уравнения найден более простой способ получения аналитических аппроксимаций $S$-матрицы реактивного рассеяния. Этот способ заключается в доказанной возможности сведения задачи об $S$-матрице коллинеарной модели перестройки к известной в квантовой механике задаче об осцилляторе с переменной частотой в поле внешней силы. Найденная связь позволяет использовать при расчете $S$-матрицы реактивного рассеяния известные аналитические представления вероятности возбуждения осциллятора через решение соответствуюшей классической задачи. Это имеет принципиальное значение для теории столкновений с перестройкой и представляет собой новый подход, устраняющий трудности использования различных, не ортогональных друг другу базисов в начальном и конечном каналах. Особый интерес представляют поиски путей обобщения этого результата на трехмерные задачи с целью использования аналитических представлений решений квантово-механических задач с гамильтонианами в виде квадратичной формы координат и импульсов.

\section{2. ПОСТАНОВКА ЗА ДАЧИ}

Будем рассматривать коллинеарную задачу трех тел $A, B, C$ с массами $m_{A}, m_{B}, m_{C}$, приводящую к трем возможным каналам рассеяния: возбуждения, перестройки и диссоциации -

$$
A+(B C)_{n} \longrightarrow\left\{\begin{array}{l}
A+(B C)_{m} \\
(A B)_{m}+C \\
A+B+C
\end{array}\right.
$$

В настояшей работе ограничимся рассмотрением матричных элементов $S_{n m}$, отвечающих каналу перестройки. Для вычисления этих матричных элементов необходимо, как известно, знание полной стационарной волновой функции $\Psi_{n}^{+}$, переходящей в асимптотическую волновую функцию $\Psi_{0 n}$ входного канала, и асимптотических волновых функций $\Psi_{0 m}$, отвечающих выходному каналу реакции. Задача состоит в нахождении аппроксимаций функции $\Psi_{n}^{+}$и вычислении матричных элементов $S_{n m}$. Основная сложность задачи рассеяния с перестройкой заключается в том, что граничные условия для функции $\Psi_{n}^{+}$формулируются с помощью двух различных наборов координат Якоби $R_{i}^{\prime}=R_{A-B C}, r_{i}^{\prime}=r_{B C} ; R_{f}^{\prime}=R_{C-A B}, r_{f}^{\prime}=r_{B A}$ во входном и выходном каналах, связанных между собой соотношениями

$$
\left(\begin{array}{c}
R_{f}^{\prime} \\
r_{f}^{\prime}
\end{array}\right)=A^{\prime}\left(\begin{array}{c}
R_{i}^{\prime} \\
r_{f}^{\prime}
\end{array}\right) ; \quad A^{\prime}=\left(\begin{array}{cc}
\frac{m_{A}}{m_{A}+m_{B}} & \frac{m_{B}\left(m_{A}+m_{B}+m_{C}\right)}{\left(m_{A}+m_{B}\right)\left(m_{B}+m_{C}\right)} \\
1 & -\frac{m_{C}}{m_{B}+m_{C}}
\end{array}\right) .
$$

С помощюю масштабного преобразования

$$
\begin{gathered}
R_{i}^{\prime}=a R_{i}, \quad r_{i}^{\prime}=r_{i} / a ; \quad R_{f}^{\prime}=c R_{f}, \quad r_{f}^{\prime}=r_{f} / c \\
a=\left(\mu_{B C} / \mu_{0}\right)^{1 / 2}, \quad c=\left(\mu_{A B} / \mu_{0}\right)^{1 / 2}, \quad \mu_{0}=\left(\frac{m_{A} m_{B} m_{C}}{m_{A}+m_{B}+m_{C}}\right)^{1 / 2}, \\
\mu_{B C}=\frac{m_{B} m_{C}}{m_{B}+m_{C}} \equiv \mu_{+}, \quad \mu_{A B}=\frac{m_{A} m_{B}}{m_{A}+m_{B}} \equiv \mu_{-},
\end{gathered}
$$




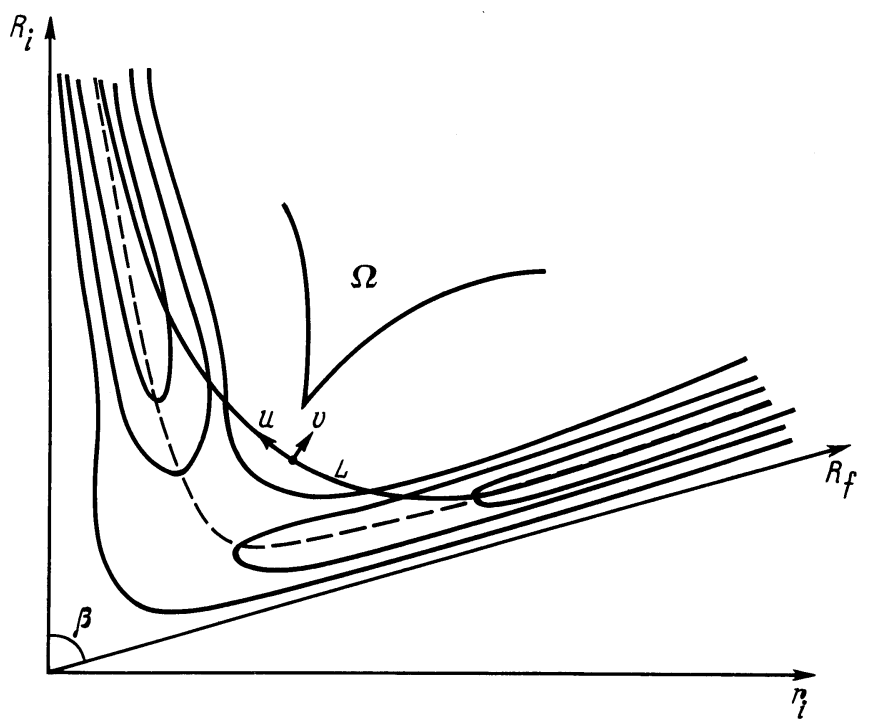

мы приводим соотношение (3) к преобразованию врашения плоскости $R_{i}, r_{i}$ на угол $\beta$

$$
\left(\begin{array}{c}
R_{f} \\
r_{f}
\end{array}\right)=A\left(\begin{array}{c}
R_{i} \\
r_{i}
\end{array}\right), \quad A=\left(\begin{array}{cc}
\cos \beta & \sin \beta \\
\sin \beta & -\cos \beta
\end{array}\right), \quad \sin \beta=a c, \quad \operatorname{tg} \beta=m_{B} / \mu_{0} .
$$

Кинетическая энергия $T$ системы трех тел в системе центра масс выражается тогда следующим образом $\left(P_{R}, p_{r}\right.$ - импульсы, канонически сопряженные координатам $\left.R, r\right)$ :

$$
T=\frac{1}{2 \mu_{0}}\left(P_{R_{i}}^{2}+p_{r_{i}}^{2}\right)=\frac{1}{2 \mu_{0}}\left(P_{R_{f}}^{2}+p_{r_{f}}^{2}\right)
$$

т.е. имеет одинаковый вид в координатах начального $\left(R_{i}, r_{i}\right)$ и конечного $\left(R_{f}, r_{f}\right)$ каналов и эквивалентна кинетической энергии точки с массой $\mu_{0}$ на плоскости, записанной в координатах начального или конечного каналов. Функция Гамильтона задачи записывается тогда в координатах начального и конечного каналов в виде

$$
H=\frac{1}{2 \mu_{0}}\left(P_{R_{i}}^{2}+p_{r_{i}}^{2}\right)+V\left(R_{i}, r_{i}\right)=\frac{1}{2 \mu_{0}}\left(P_{R_{f}}^{2}+p_{r_{f}}^{2}\right)+V\left(R_{f}, r_{f}\right)
$$

где $V$ - поверхность потенциальной энергии системы трех тел, выраженная в координатах входного и выходного каналов. Квантование системы, описьваемой гамильтонианом (6), проводится стандартным путем и приводит к двумерному оператору Лапласа, инвариантному относительно замены координат начального и конечного каналов, и потенциальной функции $V$, которая не инвариантна относительно этой замены. А именно, мы имеем следующее асимптотическое поведение $V$ в каналах (см. рисунок):

$$
V\left(R_{i}, r_{i}\right) \underset{R_{i} \rightarrow \infty}{\longrightarrow} V_{B C}\left(r_{i}\right) ; \quad V\left(R_{f}, r_{f}\right) \underset{R_{f} \rightarrow \infty}{\longrightarrow} V_{A B}\left(r_{f}\right)
$$


где $V_{B C}(r), V_{A B}(r)$ - потенциалы взаимодействия между частицами $B$ и $C$ и частицами $A$ и $B$, соответственно. Наличие различных асимптотических пределов $V$ порождает одну из трудностей теории реакций с перестройкой, связанную с использованием неортогональных базисов в начальном и конечном каналах. Один из способов преодоления этой трудности заключается в использовании криволинейных координат реакции $u, v$, отражаюших факт локализации полной волновой функции в окрестности минимума потенциальной функции $V$, по которому проходит классическая траектория изображающей точки динамической системы (путь реакции на рисунке). Координаты реакции,отличаюшиеся от пути реакции, вводятся следуюшим образом. На плоскости $R_{i}, r_{i}$ определяется некоторая кривая $L$ (рисунок), асимптотически параллельная координатным осям $R_{f}, R_{i}$. Длина дуги $u$, отсчитываемая от некоторой точки на этой кривой, является одной из координат $(-\infty<u<+\infty)$, вторая координата - расстояние $v$ по нормали к кривой $L$ в каж дой точке. Выбор кривой $L$ произволен и определяется соображениями близости кривой $L$ к пути реакции с точки зрения метрики, введенной ниже, и отсутствия неоднозначности координат $u, v$ (область $\Omega$ на рисунке) в области конфигурационного пространства, где волновая функция системы $\Psi^{+}(u, v)$ заметно отлична от нуля $[9,10]$. Вопросы выбора координат реакции неоднократно обсуждались в литературе при численном определении матричных элементов $S$-матрицы [11-14].

Уравнение Шредингера в криволинейных координатах $u, v$ имеет вид

$$
\Delta_{u, v} \Psi^{+}(u, v)+\hbar^{-2} p^{2}(u, v) \Psi^{+}(u, v)=0
$$

где $\Delta_{u, v}$ - оператор Лапласа в криволинейных координатах

$$
\Delta_{u, v}=\frac{1}{h_{u} h_{v}}\left[\frac{\partial}{\partial u}\left(\frac{h_{v}}{h_{u}} \frac{\partial}{\partial u}\right)+\frac{\partial}{\partial v}\left(\frac{h_{u}}{h_{v}} \frac{\partial}{\partial v}\right)\right], \quad h_{u}=1, \quad h_{v}=1+v / \rho,
$$

и $\rho=\rho(u)-$ радиус кривизны кривой $L$.

Полный импульс системы выражается следуюшим образом:

$$
P(u, v)=\sqrt{2 \mu_{0}(E-U(u, v))} .
$$

Граничные условия для $\Psi_{n}^{+}(u, v)$ представим в форме

$$
\begin{array}{rlrl}
\lim _{u \rightarrow-\infty} \Psi_{n}^{+}(u, v) & =\Psi_{0 n}(u, v) & & \left(R_{i} \rightarrow \infty\right), \\
\lim _{u \rightarrow \infty} \Psi_{n}^{+}(u, v) & =\sum_{m} S_{m n} \Psi_{0 m}(u, v) & \left(R_{f} \rightarrow \infty\right) .
\end{array}
$$

Здесь $S_{m n}$ имеет смысл амплитуды вероятности процесса перестройки.

\section{3. АНАЛИТИЧЕСКОЕ РЕШЕНИЕ УРАВНЕНИЯ ШРЕДИНГЕРА ДЛЯ КОЛЛИНЕАРНОЙ МОДЕЛИ ПЕРЕСТРОЙКИ В КВАЗИКЛАССИЧЕСКОМ ПРИБЛИЖЕНИИ}

При нахождении квазиклассических аппроксимаций $\Psi^{+}(u, v)$ при $\hbar \rightarrow 0$ будем исходить из следуюшего представления, в котором выделяются сильно осциллируюшая на 
кривой $L$ экспонента и более плавно меняюшаяся по координате $u$ функция $A(u, v)$ (основная идея метода параболического уравнения Фока-Леонтовича [15]):

$$
\Psi^{+}(u, v)=A(u, v) \exp \left\{i \hbar^{-1} \int_{0}^{u} d u^{\prime} P\left(u^{\prime}, 0\right)\right\} .
$$

Действительно, сушествуют по крайней мере два предельных случая, в которых решение можно получить полуаналитически (в квадратурах) - это случай квазиклассического приближения и случай волновой функции, локализованной в окрестности линии (пути реакции). Асимптотический анализ [15] показывает, что отклонение точной волновой функции от предельной (за исключением некоторых особых ситуаций, требующих специального рассмотрения и связанных с тонкими физическими эффектами) меняется медленно по сравнению с самой функцией, а поэтому имеет смысл ввести представление (12) и получить для $A(u, v)$ уравнение, которое во многих отношениях является проше исходного. Нетрудно показать [15], что модифицированная волновая функция $\Psi^{+}(u, v)$ наиболее быстро меняется в окрестности пути реакции, поэтому, действуя в духе классической теории возмушений, естественно ввести растянутую поперечную координату, позволяюшую "разрешить" эти колебания. Расстановка параметров при производных в уравнении (8) показывает, что в качестве масштабного множителя естественно принять $\hbar^{-1 / 2}$.

Использование других кривых $L$ (где, например, $P(u, 0)$ меняет знак или описывает свободные частицы в асимптотической области) позволяет описывать каналы возбуждения и диссоциации.

Подставляя (12) в (8), получаем уравнение для $A$

$$
\begin{aligned}
\frac{\partial^{2} A}{\partial v^{2}} & +\left(1+\frac{v}{\rho}\right) \frac{\partial^{2} A}{\partial u^{2}}+\frac{1}{\rho}\left(1+\frac{v}{\rho}\right) \frac{\partial A}{\partial v}+\frac{2 i P}{\hbar}\left(1+\frac{v}{\rho}\right)^{-2} \frac{\partial A}{\partial u}+ \\
& +\left(1+\frac{v}{\rho}\right)^{-1} \frac{\partial}{\partial u}\left(1+\frac{v}{\rho}\right)^{-1} \frac{\partial A}{\partial u}+\frac{i}{\hbar}\left(1+\frac{v}{\rho}\right)^{-1}\left(\frac{\partial}{\partial u}\left(1+\frac{v}{\rho}\right)\right) A+ \\
+ & \frac{p^{2}}{\hbar^{2}}\left[\frac{P^{2}(u, v)}{p^{2}}-\left(1+\frac{v}{\rho}\right)^{-2}\right] A=0 ; \quad p=P(u, 0) .
\end{aligned}
$$

Плавность изменения функции $A(u, v)$ по $u$ и ее локализация по $v$ в полосе $|v|=O\left(\hbar^{1 / 2}\right)$ дают возможность получить следующие оценки [15]:

$$
A=O(1) ; \quad \frac{\partial A}{\partial u}=O(1) ; \quad \frac{\partial A}{\partial v}=O\left(\hbar^{-1 / 2}\right) ; \quad \frac{\partial^{2} A}{\partial v^{2}}=O\left(\hbar^{-1}\right) .
$$

Сохраняя в (14) члены порядка $O\left(\hbar^{-1}\right)$ и $O\left(\hbar^{-2}\right)$, получим

$$
\begin{gathered}
A_{v v}+\frac{2 i}{\hbar} p A_{u}+\frac{i}{\hbar} p_{u} A+\frac{p^{2}}{\hbar^{2}}\left[\frac{P^{2}(u, v)}{p^{2}}-\left(1+\frac{v}{\rho}\right)^{-2}\right] A=0 \\
p_{u}=\left.\frac{\partial P(u, v)}{\partial u}\right|_{v=0}
\end{gathered}
$$


В силу локализации волновой функции (12) по $v$ можно разложить полный импульс (10) по степеням $v$, ограничиваясь членами, квадратичными по $v$,

$$
P(u, v)=p+p_{v} v+\frac{1}{2} p_{v v} v^{2}+\ldots, \quad p_{v}=\left.\frac{\partial P(u, v)}{\partial v}\right|_{v=0}, \quad p_{v v}=\left.\frac{\partial^{2} P(u, v)}{\partial v^{2}}\right|_{v=0}
$$

Уравнение (15) переписывается тогда в виде

$$
\begin{gathered}
A_{v v}+\frac{2 i}{\hbar} p A_{u}+\frac{i}{\hbar} p_{u} A+\frac{2}{\hbar} p^{2} R^{-1} v^{2} A-\hbar^{-2} K(u) v^{2} A=0 \\
R(u)=\left[\rho^{-1}(u)+\frac{p_{v}(u)}{p(u)}\right]^{-1}, \quad K(u)=-p^{2}\left(\frac{p_{v v}}{p}+\frac{p_{v}^{2}}{p}+\frac{2 p_{u}}{p \rho}\right) .
\end{gathered}
$$

Функция $R(u)$, определенная соотношением (18), имеет смысл “эффективного радиуса кривизны" кривой $L$. Отметим, что если кривая $L$ совпадает с путем реакции, то $R^{-1}(u)=0[15]$

Перейдем к исследованию уравнения (17). Для этого введем новую независимую переменную

$$
x=\hbar^{-1 / 2} \varkappa(u) v, \quad \varkappa(u)>0,
$$

где функция $\varkappa(u)$ подлежит определению. Уравнение (17) в новых переменных приобретает вид

$$
A_{x x}+\frac{2 i}{\varkappa^{2}} p A_{u}+\frac{i}{\varkappa^{2}} p_{u} A+\frac{2 p^{2}}{\varkappa^{3} R^{*}} x A+\frac{2 i \varkappa_{u}}{\varkappa^{2}} p x A_{x}-\frac{K}{\varkappa^{4}} p^{2} x^{2} A=0
$$

где $R^{*} \equiv \hbar^{1 / 2} R$.

Решение уравнения (20) представим в следуюшей форме:

$$
A(u, x)=B(u, x) \exp \left\{i x^{2} l(u)\right\}
$$

где $l(u)$ - еше одна функция, подлежащая определению. Подставляя (21) в (20), находим

$$
\begin{aligned}
B_{x x}+ & 4 i x\left(l+\frac{\varkappa_{u}}{2 \varkappa^{3}} p\right) B_{x}+\frac{2 i}{\varkappa^{2}} p B_{u}+\frac{2 p^{2}}{\varkappa^{3}} R^{*-1} x B+i\left(2 l+\frac{p_{u}}{\varkappa^{2}}\right) B- \\
& -x^{2}\left(4 l^{2}+\frac{4 \varkappa_{u}}{\varkappa^{3}} l p+\frac{2 l_{u}}{\varkappa^{2}} p+\frac{K}{\varkappa^{4}}\right) B=0 .
\end{aligned}
$$

Для определения функций $\varkappa(u)$ и $l(u)$ потребуем выполнения следуюших условий:

$$
\left\{\begin{array}{c}
l(u)+\frac{\varkappa_{u}}{2 \varkappa^{3}} p=0 \\
2 l(u)+\frac{p_{u}}{\varkappa^{2}}=0 .
\end{array}\right.
$$

Решение (23) находится в явном виде:

$$
\varkappa(u)=c p, \quad l(u)=-p_{u} / 2 c^{2} p^{2},
$$


где $c$ - постоянная, которую следует определить из асимптотических условий для $\Psi_{n}^{+}(u, v)$.

С учетом (23), (24) уравнение (22) принимает вид

$$
\begin{gathered}
B_{x x}+\frac{2 i}{c^{2} p} B_{u}-x^{2} N B+\frac{2}{c^{3} p R^{*}} x B=0, \\
N=c^{-4} p^{-4}\left(p_{u}^{2}-p p_{u u}+K\right)>0 .
\end{gathered}
$$

Введем переменную $t$ согласно соотношению

$$
t=c^{2} \int_{0}^{u} d u^{\prime} P\left(u^{\prime}, 0\right)
$$

Из (26) вытекает следуюшее асимптотическое поведение функции $t=t(u)$ :

$$
t(u) \underset{u \rightarrow \pm \infty}{\longrightarrow} \pm \infty, \quad t(u) \underset{u \rightarrow 0}{\longrightarrow} 0
$$

Воспользовавшись (27), преобразуем (25) к виду

$$
i B_{t}=-\frac{1}{2} B_{x x}+\left[\frac{1}{2} \Omega^{2}(t) x^{2}-F(t) x\right] B(x, t),
$$

где величины $\Omega, F$ определяются соотношениями

$$
\Omega(t)=N^{1 / 2}, \quad F(t)=\left(c^{3} p R^{*}\right)^{-1} .
$$

Функции $\Omega(t)$ и $F(t)$ удовлетворяют следующим условиям:

$$
\lim _{t \rightarrow \pm \infty} \Omega(t)=\Omega_{ \pm}, \quad \lim _{t \rightarrow \pm \infty} F(t)=0
$$

где $\Omega_{ \pm}$суть некоторые постоянные. Следует отметить, что в случае, когда кривая $L$ является путем реакции, $F(t)=0$.

Таким образом, последовательными преобразованиями коллинеарную задачу о перестройке удается свести к одномерной нестационарной задаче о квантовом осцилляторе с переменной частотой $\Omega(t)$ в поле внешней силы $F(t)$, которая хорошо исследована в литературе (см., например, [16]). Учитывая специфику исходной задачи, необходимо привести основные результаты применительно к нашему случаю.

Пусть $F(t)=0$. Будем искать решение $(28)$ в виде

$$
B(x, t)=\sigma^{-1 / 2}(t) \exp \{-i \chi(x, t)\} D(y, \tau),
$$

где $y=x / \sigma(t), \tau=\gamma(t) / \Omega_{-}$, а функции $\sigma(t)$ и $\gamma(t)$ подлежат определению.

Подставляя (30) в (28), имеем

$$
\begin{gathered}
\chi(x, t)=-\dot{\sigma} x / 2 \sigma \\
i \frac{\dot{\gamma}}{\Omega_{-}} \frac{\partial D}{\partial \tau}=-2 \sigma^{-2} \frac{\partial^{2} D}{\partial y^{2}}+\frac{1}{2}\left[\Omega^{2}(t)+\left(\frac{\dot{\sigma}}{\sigma}\right)^{2}+\frac{d}{d t} \frac{\dot{\sigma}}{\sigma}\right] x D .
\end{gathered}
$$


Потребуем выполнения следующих условий:

$$
\dot{\gamma}=\Omega_{-} / \sigma^{2}, \quad \ddot{\sigma} / \sigma+\Omega^{2}(t)=\dot{\gamma}^{2} .
$$

При этом (32) принимает вид

$$
i D_{\tau}=-\frac{1}{2} D_{y y}+\frac{1}{2} \Omega_{-}^{2} y^{2} D .
$$

Для $D(y, \tau)$ имеем решение

$$
D(y, \tau)=\left(\frac{1}{2^{n} n !} \sqrt{\frac{\Omega_{-}}{\pi}}\right)^{1 / 2} \exp \left(-i E_{n} \tau-\frac{1}{2} \Omega_{-} y^{2}\right) H_{n}\left(\sqrt{\Omega_{-}} y\right),
$$

где $H_{n}(z)$ - полином Эрмита,

$$
E_{n}=(n+1 / 2) \Omega_{-}, \quad \tau=\int_{-\infty}^{t} d t^{\prime} / \sigma\left(t^{\prime}\right) .
$$

Таким образом, для решения (28) при $F(t)=0$ достаточно найти функции $\sigma(t)$ и $\gamma(t)$. Перепишем (33) следуюшим образом:

$$
\ddot{\sigma}(t)+\Omega^{2}(t) \sigma(t)=\Omega_{-}^{2} / \sigma^{3}(t) .
$$

Граничные условия для $\sigma(t)$ и $\gamma(t)$ вытекают из требования существования при $t \rightarrow-\infty$ стационарных решений $(28)$ и имеют вид

$$
\lim _{t \rightarrow-\infty} \sigma(t)=1, \quad \gamma(t) \underset{t \rightarrow-\infty}{\sim} \Omega_{-} t .
$$

Общее решение уравнения (28) записывается таким образом:

$$
\sigma(t)=\left[\sum_{i, k=1}^{2} a_{i k} \xi_{i}(t) \xi_{k}(t)\right]^{1 / 2}
$$

где $\xi_{1}(t)$ и $\xi_{2}(t)$ - два линейно независимых решения однородного уравнения

$$
\ddot{\xi}(t)+\Omega^{2}(t) \xi(t)=0
$$

и $a_{i j}(i, j=1,2)$ - произвольные комплексные постоянные, удовлетворяюшие условиям

$$
W^{2} \operatorname{det}\left\|a_{i j}\right\|=1, \quad a_{i j}=a_{j i} ; \quad W \equiv \xi_{1} \dot{\xi}_{2}-\xi_{2} \dot{\xi}_{1} .
$$

В качестве линейно независимых решений $\xi_{1,2}$ выберем

$$
\xi_{1}(t)=\xi(t), \quad \xi_{2}(t)=\xi^{*}(t) .
$$


Граничные условия для $\xi(t)$ полностью определяют решение (40), а следовательно, и функции $\sigma(t)$ и $\gamma(t)$. Положим

$$
\xi(t) \underset{t \rightarrow-\infty}{=} \widetilde{C}_{1} e^{i \Omega_{-} t}+\widetilde{C}_{2} e^{-i \Omega_{-} t}, \quad \widetilde{C}_{1}=\left|\widetilde{C}_{1}\right| e^{i \tilde{\beta}_{1}}, \quad \widetilde{C}_{2}=\left|\widetilde{C}_{2}\right| e^{i \tilde{\beta}_{2}},
$$

где $\left|\widetilde{C}_{i}\right|, \tilde{\beta}_{i}(i=1,2)$ - произвольные вешественные постоянные. Подставляя (43) в (39) и учитывая (41), путем простых вычислений можно показать, что условия (38) вьполняются при выполнении следующих требований:

$$
\left|\widetilde{C}_{2}\right|=0, \quad \tilde{\beta}_{i}=0, \quad\left|\widetilde{C}_{1}\right|=1 .
$$

Таким образом, решение (30) уравнения (28) при $F(t)=0$ определяется решениями уравнения (40) с граничными условиями

$$
\xi(t) \underset{t \rightarrow-\infty}{\sim} e^{i \Omega_{-} t}, \quad \xi(t) \underset{t \rightarrow \infty}{\sim} C_{1} e^{i \Omega_{+} t}-C_{2} e^{-i \Omega_{+} t},
$$

где $C_{i}$ - произвольные комплексные постоянные, между которыми сушествует очевидное соотношение

$$
\left|C_{1}\right|^{2}-\left|C_{2}^{2}\right|=\Omega_{-} / \Omega_{+}
$$

Введем величину $\vartheta$,

$$
\vartheta=\left|C_{2} / C_{1}\right|^{2}
$$

которая при заданном отношении $\Omega_{+} / \Omega_{-}$определяет модули коэффициентов $C_{1}$ и $C_{2}$,

$$
C_{1}=e^{i \delta_{1}} \sqrt{\Omega_{-} / \Omega_{+}}(1-\vartheta)^{-1 / 2}, \quad C_{2}=e^{i \delta_{2}} \sqrt{\Omega_{-} / \Omega_{+}} \sqrt{\vartheta /(1-\vartheta)} .
$$

Величина $\vartheta$ связана с коэффициентом отражения от одномерного потенциального барьера. Образуем из $\xi(t)$ и $\xi^{*}(t)$ линейную комбинацию $\xi_{1}(t)$ со следующими свойствами:

$$
\xi_{1}(t)=\xi(t)+R \xi^{*}(t)= \begin{cases}e^{i \Omega_{-} t}+R e^{-i \Omega_{-} t}, & t \rightarrow-\infty \\ D e^{i \Omega_{+} t}, & t \rightarrow \infty .\end{cases}
$$

Видно, что $\xi_{1}(t)$ совпадает с волновой функцией одномерного уравнения Шредингера, имеюшего вид (40) . Тогда коэффициенты $R$ и $D$ имеют смысл амплитуд отраженной и прошедшей волн [17]. Сравнивая (49) с (45), легко получить

$$
C_{1}=D /\left(1-|R|^{2}\right), \quad C_{2}=R D^{*} /\left(1-|R|^{2}\right), \quad \vartheta=|R|^{2} .
$$

Это обстоятельство позволяет использовать для определения $\vartheta$ известные результаты квантовой механики, полученные, например, в рамках метода ВКБ.

Когда на осциллятор действует внешняя сила (кривая $L$ не является путем реакции), задачу можно свести к случаю отсутствия внешней силы с помошью подстановки [18]

$$
B(x, t)=\exp \left\{i\left(S_{\mathrm{cl}}(t)+\dot{\eta} \bar{x}\right)\right\} \varphi(\bar{x}, t), \quad \bar{x}=x-\eta(t) .
$$

Подставляя (51) в (28), для функции $\varphi(t)$ получим

$$
i \varphi_{t}=-\frac{1}{2} \varphi_{\bar{x} \bar{x}}+\frac{1}{2} \Omega^{2} \bar{x}^{2} \varphi+\left(\eta_{t t}+\Omega^{2} \eta-F\right) \bar{x} \varphi+\left(\dot{S}_{\mathrm{cl}}-\frac{1}{2}\left(\eta_{t}^{2}-\Omega^{2} \eta^{2}\right)-F \eta\right) \varphi .
$$


Для сведения задачи к предыдущему случаю потребуем вьполнения условий

$$
\begin{gathered}
\eta_{t t}+\Omega^{2}(t) \eta=F(t), \quad \eta(-\infty)=\eta_{t}(-\infty)=0 \\
S_{\mathrm{cl}}=\int_{-\infty}^{t} d t^{\prime}\left(\frac{1}{2} \eta_{t^{\prime}}^{2}-\frac{1}{2} \Omega^{2}\left(t^{\prime}\right) \eta^{2}+F \eta\right)
\end{gathered}
$$

Тем самым неизвестные в (51) определены. Функцию $\eta(t)$, являюшуюся решением (53), можно выразить через решение $\xi(t)$ уравнения (40):

$$
\eta(t)=\left(2 \Omega_{-}\right)^{-1 / 2}\left(\xi(t) d^{*}(t)+\xi^{*}(t) d(t)\right) .
$$

Здесь введена важная для дальнейшего функция $d(t)$ :

$$
\begin{aligned}
d(t) & =i /\left(2 \Omega_{-}\right)^{1 / 2} \int_{-\infty}^{t} d t^{\prime} \xi\left(t^{\prime}\right) F\left(t^{\prime}\right) \\
d & =\lim _{t \rightarrow \infty} d(t) \equiv(\nu)^{1 / 2} e^{i \beta}
\end{aligned}
$$

Учитывая (30), (31), можно найти общее решение уравнения (28)

$$
B(x, t)=(\sigma(t))^{-1 / 2} \exp \left\{i\left[S_{\mathrm{cl}}+\dot{\eta}(x-\eta)+\frac{\dot{\sigma}}{2 \sigma}(x-\eta)^{2}\right]\right\} D\left(\frac{x-\eta}{\sigma}, \tau\right) .
$$

Решение уравнения Шредингера (8), описываюшее эволюцию коллинеарной системы трех тел в канале с перестройкой, несложно теперь получить, объединяя (12), (21) и $(58)$,

$$
\begin{aligned}
\Psi_{n}^{+}(x, t)= & (\sigma)^{-1 / 2} \exp \left(i \frac{\widetilde{S}}{\hbar}\right)\left[(2 n !)^{-1 / 2} \sqrt{\frac{\Omega_{-}}{\pi}}\right]^{1 / 2} \times \\
& \times \exp \left(-\frac{\Omega_{-}}{2}\left(\frac{x-\eta}{\sigma}\right)^{2} H_{n}\left(-\frac{\left(\Omega_{-}\right)^{1 / 2}}{\sigma}(x-\eta)\right),\right.
\end{aligned}
$$

где $\widetilde{S}$ имеет смысл классического действия системы трех тел, учитывающего вкладпримыкающих траекторий в токовой трубке:

$$
\begin{aligned}
\widetilde{S}= & S_{\mathrm{cl}}^{*}-\hbar\left[(n+1 / 2) \Omega_{-} \int_{-\infty}^{t} d t^{\prime} / \sigma^{2}\left(t^{\prime}\right)+(\dot{\eta}-\dot{p} \eta / p)(x-\eta)+\right. \\
& \left.+\frac{1}{2}(\dot{\sigma} / \sigma-\dot{p} / p)(x-\eta)^{2}-\frac{\dot{p}}{2 p} \eta^{2}\right], \quad S_{\mathrm{cl}}^{*}=c^{-2} t+\hbar S_{\mathrm{cl}}(t) .
\end{aligned}
$$

Для определения постоянной $c$ в соотношении (24) необходимо перейти к пределу $t \rightarrow-\infty$ в уравнении (59). Тогда из условия

$$
\lim _{t \rightarrow-\infty} \Psi_{n}^{+}(x, t)=\Psi_{0 n}(x, t)
$$


легко найти

$$
c^{2}=\frac{\hbar}{p_{-}}\left(\mu_{0} / \mu_{-}\right)^{1 / 2}, \quad p=\left.\lim _{u \rightarrow-\infty} P(u, v)\right|_{v=0} .
$$

\section{4. ВЫЧИСЛЕНИЕ ВЕРОЯТНОСТИ НАДБАРЬЕРНОГО РЕАКТИВНОГО ПЕРЕХОДА}

Конечной целью задачи является нахождение вероятности реактивных переходов путем вычисления матричных элементов $S_{m n}$. Эта задача решается следуюшим образом. В силу (59) мы знаем квазиклассическое приближение к полной волновой функции $\Psi^{+}(x, t)$ при любом $t$, в том числе и при $t \rightarrow \pm \infty$. Коэффициенты разложения этой функции по асимптотическим волновым функциям $\Psi_{0 m}(x, t)$ в конечном канале суть искомые матричные элементы. Этот способ вычисления коэффициентов разложения можно упростить, введя производящую функцию

$$
\Psi(z, x, t)=\sum_{n=0}^{\infty} \frac{z^{n}}{(n !)^{1 / 2}} \Psi_{n}^{+}(x, t),
$$

где $z$ - некоторая вспомогательная комплексная переменная.

Подставляя в $(63) \Psi_{n}^{+}(x, t)$ из $(59)$ и производя суммирование, получим

$$
\begin{aligned}
& \Psi(z, x, t)=(\sigma(t))^{-1 / 2}\left(\Omega_{-} / \pi\right)^{1 / 4} \exp \left\{-\frac{1}{2}\left[-i \frac{\dot{\sigma}}{\sigma}(x-\eta)^{2}-\frac{\dot{p}}{p}(x-\eta)^{2}-\right.\right. \\
& \left.\left.-\frac{2\left(2 \Omega_{-}\right)^{1 / 2}}{\sigma}(x-\eta) z+z^{2} e^{-2 i \gamma}\right]+i\left[(\dot{\eta}-\dot{p} \eta / p)(x-\eta)-\frac{\dot{p}}{2 p} \eta^{2}+S_{\mathrm{cl}}^{*} / \hbar\right]\right\} .
\end{aligned}
$$

Аналогично вычисляется производящая функция асимптотического состояния при $t \rightarrow \infty$ :

$$
\begin{aligned}
\Psi_{0}(z, x, t)= & (\Omega / \pi)^{1 / 4} \exp \left\{-\frac{1}{2}\left(\Omega_{+} x^{2}-2\left(2 \Omega_{+}\right)^{1 / 2} z x e^{-i \Omega_{+} t}+\right.\right. \\
& \left.\left.+z^{2} e^{-2 i \Omega_{+} t}+i \Omega_{+} t\right)+i c^{2} t / \hbar\right\} .
\end{aligned}
$$

Рассмотрим интеграл

$$
I\left(z_{1}, z_{2}, t\right)=\int_{-\infty}^{\infty} d x \Psi_{0}^{*}\left(z_{1}, x, t\right) \Psi\left(z_{2}, x, t\right)
$$

При $t \rightarrow \infty$ этот интеграл совпадает с производящей функцией матричных элементов $S_{n m}$,

$$
\lim _{t \rightarrow \infty} I\left(z_{1}, z_{2}, t\right)=J\left(z_{1}, z_{2}\right)=\sum_{n, m=0}^{\infty} \frac{z^{m} z^{n}}{(m ! n !)^{1 / 2}} S_{n m}
$$

Следуя работе [19], можно получить для $J\left(z_{1}, z_{2}\right)$ следуюшее выражение:

$$
\begin{aligned}
J\left(z_{1}, z_{2}\right)= & (1-\vartheta)^{1 / 4} \exp \left\{-\frac{\nu}{2}(1-\sqrt{\vartheta} \cos 2 \varphi)+\right. \\
& +\frac{1}{2}\left[\sqrt{\vartheta}\left(z_{2}^{2}-z_{1}^{2}\right)+2 \sqrt{1-\vartheta} z_{1} z_{2}\right]+ \\
& \left.+\sqrt{\vartheta}\left[\sqrt{1-\vartheta} e^{-i \varphi} z_{1}-\left(e^{i \varphi}-\sqrt{\vartheta} e^{-i \varphi}\right) z_{2}\right]\right\} .
\end{aligned}
$$


При получении (68) учтены условия (45) и (53), а также опушены несушественные для вычисления вероятностей переходов фазовые множители у $z_{1}$ и $z_{2}$.

Вычисление матричных элементов $S_{n m}$ сводится к разложению функции $J\left(z_{1}, z_{2}\right)$ в ряд Тейлора [16]. Приведем окончательное выражение для вероятности перестройки:

$$
W_{m n}=\sqrt{\frac{1-\vartheta}{m ! n !}}\left|H_{m n}\left(y_{1}, y_{2}\right)\right|^{2} \exp \{-\nu(1-\sqrt{\vartheta} \cos 2 \varphi)\}
$$

Здесь $H_{m n}\left(y_{1}, y_{2}\right)$ суть полиномы Эрмита от двух переменных [20]:

$$
y_{1}=\sqrt{\nu(1-\vartheta)} e^{i \varphi}, \quad y_{2}=-\sqrt{\nu}\left(e^{-i \varphi}-e^{i \varphi}\right), \quad \varphi=\left(\delta_{1}+\delta_{2}\right) / 2-\beta,
$$

а параметры $\vartheta, \nu, \beta, \delta_{1}$ и $\delta_{2}$ определены соотношениями $(47),(57)$ и (48), соответственно.

В случае отсутствия внешней силы $\nu=0$ и выражение для $W_{m n}$ можно упростить:

$$
\widetilde{W}_{m n}=\frac{n_{<} !}{n_{>} !} \sqrt{1-\vartheta}\left|P_{|m+n| / 2}^{|n-m| / 2}(\sqrt{1-\vartheta})\right|^{2} .
$$

Здесь $n_{<}=\min (m, n), P_{n}^{m}(x)-$ присоединенная функция Лежандра [19].

Таким образом, если кривая $L$ является путем реакции, вероятности перестройки полностью определяются одним параметром $\vartheta$, для нахождения которого необходимо решить уравнение (40) с граничными условиями (45).

\section{5. ЗАКЛЮЧЕНИЕ}

Полученные результаты дают основу для нового подхода к проблеме коллинеарных реакций, альтернативного традиционным методам сильной связи каналов, классической $S$-матрицы, и поэтому представляют большой интерес как для численных расчетов, так и для возможных обобшений.

Таким образом, в квазиклассическом приближении при определенном выборе кривой $L$ удается получить аналитические аппроксимации вероятности надбарьерного реактивного перехода для произвольной потенциальной функции $V$. Формализм позволяет находить ангармонические поправки, а за счет выбора $L$ можно строить аналитические аппроксимации вероятностей перестройки, а также возбуждения и диссоциации, учитьвающие влияние туннельных переходов, резонансных точек, используя известные численные данные траекторных расчетов для коллинеарной задачи трех тел. Обсуждению этих вопросов будут посвящены наши следующие сообщения.

Авторы благодарят Российский фонд фундаментальных исследований за финансирование проекта (грант № 95-01-00491), в рамках которого была выполнена эта работа.

\section{Список литературы}

[1] Miller W.H. Dynamics of molecular collisions. N.Y. - London: Plenum Press, 1976.

[2] Мизер В.О., Фейербах С., Роттенбергер М. Вычислительные методы в физике атомных и молекулярных столкновений. М.: Мир, 1974.

[3] Ломакин Л.А., Ошеров В.И., Поляков О.Н. // Хим. физ. 1982. №5. С. 594-598.

[4] Теоретические проблемы химической физики. М.: Мир, 1969.

[5] Геворкян А.С. // ДАН Арм. СССР. 1983. Т. 7. № 5. С. 219-224.

[6] Богданов А.В., Геворкян А.С., Горбачев Ю.Е., Дубровский Г.В. // Вестник ЛГУ. 1983. № 22 . C. $80-82$. 
[7] Богданов А.В., Горбачев Ю.Е., Геворкян А.С., Дубровский Г.В. Квазиклассическое интегральное представление $T$-оператора для процессов с перестройкой в рамках коллинеарной модели: Препринт № 998. Л.: ФТИ АН СССР, 1986.

[8] Денисенко А.И., Литвиненко С.А., Соколов В.И. Анализ элементарных физических процессов, протекающих при формировании структуры диэлектрик-полупроводник: Препринт № 1469. Л.: ФТИ АН СССР, 1990.

[9] Marcus R.A.// J. Chem. Phys. 1966. V. 45. P. 4493-4498.

[10] Marcus R.A. // J. Chem. Phys. 1968. V. 49. P. 2610-2617.

[11] Pancin C.C., Light J. // J. Chem. Phys. 1969. V. 51. P. 1701-1704.

[12] Miller G., Light J. // J. Chem. Phys. 1971. V. 54. P. 1635-1642.

[13] Miller G., Light J. // J. Chem. Phys. 1971. V. 54. P. 1643-1648.

[14] Light J. // Adv. Chem. Phys. 1971. V. 19. P. 1-22.

[15] Бабич B.M., Булдырев В.С. Асимптотические методы в задачах дифракции коротких волн. М.: Наука, 1972.

[16] Базь А.И., Зельдович Я.Б., Переломов А.М. Рассеяние, реакции и распады в нерелятивистской квантовой механике. М.: Наука, 1976.

[17] Ландау Л.Д., Лифиич, Е.М. Квантовая механика. М.: Наука, 1976.

[18] Husimi K. // Progr. Theor. Phys. 1953. V. 9. P. 381-394.

[19] Попов В.С., Переломов А.М. // ЖЭТФ. 1969. Т. 56. С. 1355-1361.

[20] Переломов A.M., Попов B.С. // ТМФ. 1970. Т. 3. С. 377-381.

Институт межфозных взаимодействий,

Технический университет

Поступила в редакцию

12.VII.1994 г.

\section{A. V. Bogdanov, A.S. Gevorkyan, A. I. Denisenko, G. V. Dubrovsky \\ QUASI CLASSICAL ANALYTICAL APPROXIMATION TO THE $S$-MATRIX OF COLLINEAR REARRANGEMENT REACTION}

Collinear problem of three-particle rearrangement reaction in the framework of the quasi classical method of Fock-Leontovich parabolic equation is reduced to the quantum problem of the one-dimensional harmonic oscillator with variable frequency in an external force field. This allows one to express the $S$-matrix elements responsible for the rearrangement channel via analytical solution of the oscillator model by using the parameters of the linear configuration of the three particles. 